\title{
Genomic and Phenotypic Analysis of Multidrug-Resistant Acinetobacter baumannii Clinical Isolates Carrying Different Types of CRISPR/Cas Systems
}

\author{
Marina Tyumentseva ${ }^{1,+}$, Yulia Mikhaylova ${ }^{1,+}{ }^{\mathbb{D}}$, Anna Prelovskaya ${ }^{1}$, Aleksandr Tyumentsev ${ }^{1}$ (D), \\ Lyudmila Petrova ${ }^{2}$, Valeria Fomina ${ }^{2}$, Mikhail Zamyatin ${ }^{2} \mathbb{D}$, Andrey Shelenkov ${ }^{1, *(D)}$ and Vasiliy Akimkin ${ }^{1}$ \\ 1 Central Research Institute of Epidemiology, Novogireevskaya str., 3a, 111123 Moscow, Russia; \\ tyumentseva@cmd.su (M.T.); mihailova@cmd.su (Y.M.); prelovskaya@cmd.su (A.P.); \\ tymencev@cmd.su (A.T.); vgakimkin@yandex.ru (V.A.) \\ 2 National Medical and Surgical Center named after N.I. Pirogov, Nizhnyaya Pervomayskaya str., 70, \\ 105203 Moscow, Russia; lutix85@yandex.ru (L.P.); med_2006@mail.ru (V.F.); mnz1@yandex.ru (M.Z.) \\ * Correspondence: fallandar@gmail.com \\ + These authors contributed equally.
}

Citation: Tyumentseva, M.; Mikhaylova, Y.; Prelovskaya, A.; Tyumentsev, A.; Petrova, L.; Fomina, V.; Zamyatin, M.; Shelenkov, A.; Akimkin, V. Genomic and Phenotypic Analysis of Multidrug-Resistant Acinetobacter baumannii Clinical Isolates Carrying Different Types of CRISPR/Cas Systems. Pathogens 2021, 10, 205. https://doi.org/10.3390/ pathogens10020205

Academic Editor: Luisa De Martino

Received: 6 January 2021

Accepted: 10 February 2021

Published: 13 February 2021

Publisher's Note: MDPI stays neutral with regard to jurisdictional claims in published maps and institutional affiliations.

Copyright: (C) 2021 by the authors Licensee MDPI, Basel, Switzerland. This article is an open access article distributed under the terms and conditions of the Creative Commons Attribution (CC BY) license (https:/ / creativecommons.org/licenses/by/ $4.0 /)$.

\begin{abstract}
Acinetobacter baumannii is an opportunistic pathogen being one of the most important causative agents of a wide range of nosocomial infections associated with multidrug resistance and high mortality rate. This study presents a multiparametric and correlation analyses of clinical multidrug-resistant $A$. baumannii isolates using short- and long-read whole-genome sequencing, which allowed us to reveal specific characteristics of the isolates with different CRISPR/Cas systems. We also compared antibiotic resistance and virulence gene acquisition for the groups of the isolates having functional CRISPR/Cas systems, just CRISPR arrays without cas genes, and without detectable CRISPR spacers. The data include three schemes of molecular typing, phenotypic and genotypic antibiotic resistance determination, as well as phylogenetic analysis of full-length cas gene sequences, predicted prophage sequences and CRISPR array type determination. For the first time the differences between the isolates carrying Type I-F1 and Type I-F2 CRISPR/Cas systems were investigated. A. baumannii isolates with Type I-F1 system were shown to have smaller number of reliably detected CRISPR arrays, and thus they could more easily adapt to environmental conditions through acquisition of antibiotic resistance genes, while Type I-F2 A. baumannii might have stronger "immunity" and use CRISPR/Cas system to block the dissemination of these genes. In addition, virulence factors abaI, abaR, bap and bauA were overrepresented in A. baumannii isolates lacking CRISPR/Cas system. This indicates the role of CRISPR/Cas in fighting against phage infections and preventing horizontal gene transfer. We believe that the data presented will contribute to further investigations in the field of antimicrobial resistance and CRISPR/Cas studies.
\end{abstract}

Keywords: Acinetobacter baumannii; whole genome sequencing; antibiotic resistance; MDR; CRISPR/ Cas systems

\section{Introduction}

Acinetobacter baumannii is an important opportunistic pathogen responsible for a wide range of hospital-acquired infections (HAI), and is associated with respiratory infections, bacteremia, meningitis, and wound infections [1]. The percentage of carbapenem- and multidrug-resistant strains causing nosocomial outbreaks in various regions of the world is growing exponentially [2]. Recently, the World Health Organization has included carbapenem-resistant $A$. baumannii in the Global List of critical priority level for scientific research and the development of new antibiotics [3].

The main features of $A$. baumannii are the intrinsic multidrug resistance; the ability to form biofilms (both on the tissues of a living organism and on polymeric materials used in 
medicine); the presence of a signaling system "quorum-sensing", which makes it possible to enhance the protection of bacteria against antibiotics, disinfectants, and the human immune system [4]. A. baumannii is characterized by high epidemic potential and quickly forms hospital clones. For the epidemiological surveillance of this important species, two multilocus sequence typing schemes (MLST), usually referenced as Oxford and Pasteur and showing different levels of resolution, have been established [5,6].

Clustered regularly interspaced short palindromic repeat (CRISPR) arrays and CRISPRassociated genes (cas) constitute bacterial adaptive immune systems and function as a variable genetic element [7]. CRISPRs are bacterial loci whose dynamic nature has allowed them to become ideal targets for molecular subtyping. Each CRISPR/Cas locus includes a strain-specific array of spacers that has expanded and diversified over time. Due to their dynamic nature, comparative analysis of the spacer arrays has successfully been used for subtyping isolates from several Gram-positive and Gram-negative bacteria, including $M y$ cobacterium tuberculosis, Yersinia pestis and the plant pathogen Erwinia amylovora [8]. Arrays of spacers were found to be highly polymorphic in Salmonella, and a strong correlation was detected between polymorphisms in the arrays and the serotypes [8,9]. Multiple reports have suggested that CRISPR/Cas systems may play a major role in controlling horizontal gene transfer and, consequently, the dynamics of antibiotic resistance gene acquisition in Pseudomonas aeruginosa $[10,11]$.

Two CRISPR/Cas systems have recently been found in the genomes of several $A$. baumannii strains $[12,13]$. Studies have reported that trailer end spacers of CRISPR are generally conserved among different isolates and can be used to anchor clusters and detect common ancestors of the arrays and, probably, of the isolates themselves [14]. More and more data are accumulating indicating that the role of CRISPR/Cas is not limited to adaptive immunity. It has been shown that these systems regulate the expression of many bacterial genes affecting the virulence of pathogenic bacteria and group behavior, and also participate in DNA repair and accelerate the evolution of genomes [15].

A. baumannii is one of the most common causative agents of HAI infections in Russia [16]. In the present study, we provide the comprehensive data based on second- and third-generation (long-read) sequencing for 12 clinical isolates of $A$. baumannii obtained from Moscow multidisciplinary medical center. We used Pasteur MLST typing scheme complemented by capsular (KL) and oligosaccharide (OCL) loci. We also explored genes associated with antimicrobial resistance and conducted sequenced-based analysis of CRISPR arrays and CRISPR/Cas systems. The abundance of the latter was shown to be associated with antibiotic susceptibility in Streptococcus pyogenes, Escherichia coli and Klebsiella pneumoniae [17-19]. Comparative analysis allowed determining the phylogenetic relationship between the cas 1 and other CRISPR/Cas loci from the isolates studied and a collection of publicly available global $A$. baumannii strains. Additionally, we assessed specific features of A. baumannii isolates having different types of CRISPR/Cas systems and compared the groups of the isolates with and without functional CRISPR/Cas systems.

\section{Results}

\subsection{Isolate Typing}

MLST is commonly used as a method of choice for epidemiological surveillance of pathogenic bacteria, if such a scheme exists for the species under study. Additional typing of A. baumannii may be performed by using capsule synthesis loci (K-loci) [20] or lipooligosaccharide outer core loci (OCL) [21]. The results of the isolate typing are presented in Table 1 . The isolates belonged to eight different sequence types according to Pasteur MLST scheme. It was chosen for typing since, according to the Oxford scheme, five isolate possessed undetectable sequence types (STs) with duplicated $g d h B$ locus. ST2 was found in four isolates, but they have different KL types that was in line with our goal to cover the representative population of all hospital isolates. It was also interesting that two isolates, CriePir306 and CriePir308, were obtained from the same patient (A9), but had different STs, thus indicating simultaneous infection by multiple strains. The isolate 
CriePir307 possessed novel ST, which allelic profile was cpn60 (8), fusA (1), gltA (16), pyrG (3), recA (6), rplB (2), rpoB (3). We have submitted this isolate to the Institut Pasteur MLST system (http:/ / bigsdb.pasteur.fr, accessed on 20 December 2020), and new profile ST1487 was assigned to this allele combination.

Table 1. The origin and typing of clinical A. baumannii isolates studied.

\begin{tabular}{|c|c|c|c|c|c|c|c|}
\hline Sample ID & Patient Code & Isolation Date & Clinical Department & Locus & MLST & OCL-Type & KL-Type \\
\hline CriePir33 & A1 & 03.05.2017 & Traumatology & Wound & ST78 & OCL1 & KL3 \\
\hline CriePir87 & $\mathrm{A} 2$ & 04.07.2017 & Surgery & Soft tissue abscess & ST2 & OCL1 & KL33 \\
\hline CriePir158 & A3 & 13.11.2017 & Therapy & Sputum & ST45 & OCL1 & KL49 \\
\hline CriePir168 & A4 & 03.12.2017 & ICU anesthesiology & Urine & ST1 & OCL1 & KL17 \\
\hline CriePir221 & A5 & 21.05.2018 & ICU anesthesiology & CVC & ST78 & OCL1 & KL2 \\
\hline CriePir298 & A6 & 10.09.2019 & Rehabilitation of CNS & Urine & ST25 & OCL6 & KL116 \\
\hline CriePir299 & A7 & 10.04.2019 & Neurosurgery & Wound & ST2 & OCL1 & KL2 \\
\hline CriePir302 & A8 & 21.06.2019 & Surgery & Abdominal abscess & ST2 & OCL1 & KL9 \\
\hline CriePir306 & A9 & 05.08.2019 & ICU anesthesiology & BAL & ST15 & OCL7 & KL9 \\
\hline CriePir307 & A10 & 29.06.2019 & ICU anesthesiology & BAL & ST1487* & OCL2 & KL45 \\
\hline CriePir308 & A9 & 22.08.2019 & ICU anesthesiology & CVC & ST2 & OCL7 & KL9 \\
\hline CriePir309 & A11 & 31.08.2019 & Rehabilitation of CNS & Urine & ST911 & OCL6 & KL14 \\
\hline
\end{tabular}

* Novel sequence type found by us is shown in bold; ICU-intensive care unit; CVC-central venous catheter; BAL-bronchoalveolar lavage; the isolates sequenced on MinION are filled with a gray color.

In addition, the isolates were assigned to nine different KL-types and four OCLtypes. The most prevalent KL-types were KL9 and KL2, which were totally revealed in about $36 \%$ of the isolates from NCBI WGS database [21]. Interestingly, CriePir298 had a recently described KL116 type [22], which is rather rare [21]. The most frequent OCL was OCL1, which was also the most common type in NCBI WGS database [21]. In general, currently $92 \mathrm{KL}$ and $12 \mathrm{OCL}$ types are available, so that our dataset shows high variability in lipooligosaccharide loci types and relatively low variability in capsule synthesis loci types.

It is worth noting that KL-type provides additional classification narrowing in comparison to sequence type alone. For example, CriePir33 forms standalone clade since it possesses the KL-type (KL3) different from its neighbors' type (KL2), although they share the same ST (ST78). The same is observed for CriePir87-it has KL33, while its neighbors with identical ST (ST2) are characterized by KL2. Thus, such a hybrid typing scheme that we have recently applied for K. pneumoniae [23], proved to be applicable for precise distinguishing of the isolates in clinical settings, which is an important task in epidemiological surveillance and infection prevention.

Additional typing performed using cgMLST loci complied well with MLST and KL analysis. cgMLST profiles for the isolates studied are presented in Table S1, and cgMLSTbased tree is shown in the Figure S11.

\subsection{Antibiotic Susceptibility Testing and Antimicrobial Resistance Gene Identification}

The results of phenotypic analysis are presented in Figure 1. Four isolates (CriePir33, CriePir87, CriePir221, and CriePir298) were resistant to all antibiotics tested. The isolate CriePir299 was susceptible to trimethoprim/sulfamethoxazole only. CriePir168 was susceptible to three antibiotics-amikacin, tobramycin, and trimethoprim/sulfamethoxazole, while other two isolates (CriePir302 and CriePir306) were susceptible to meropenem, imipenem and trimethoprim/sulfamethoxazole in addition to tobramycin. The isolates CriePir307 and CriePir309 were susceptible to all antibiotics tested.

We examined the distribution of acquired antibiotic resistance genes among the clinical isolates studied. The results of resistance determinant annotation are presented in Figure 1. The spectrum of identified resistance determinants included gene clusters providing resistance to aminoglycosides, beta-lactams, chloramphenicol and sulfonamides. Some isolates were characterized by the presence of genes determining resistance to rifampicin, erythromycin and tetracycline. 


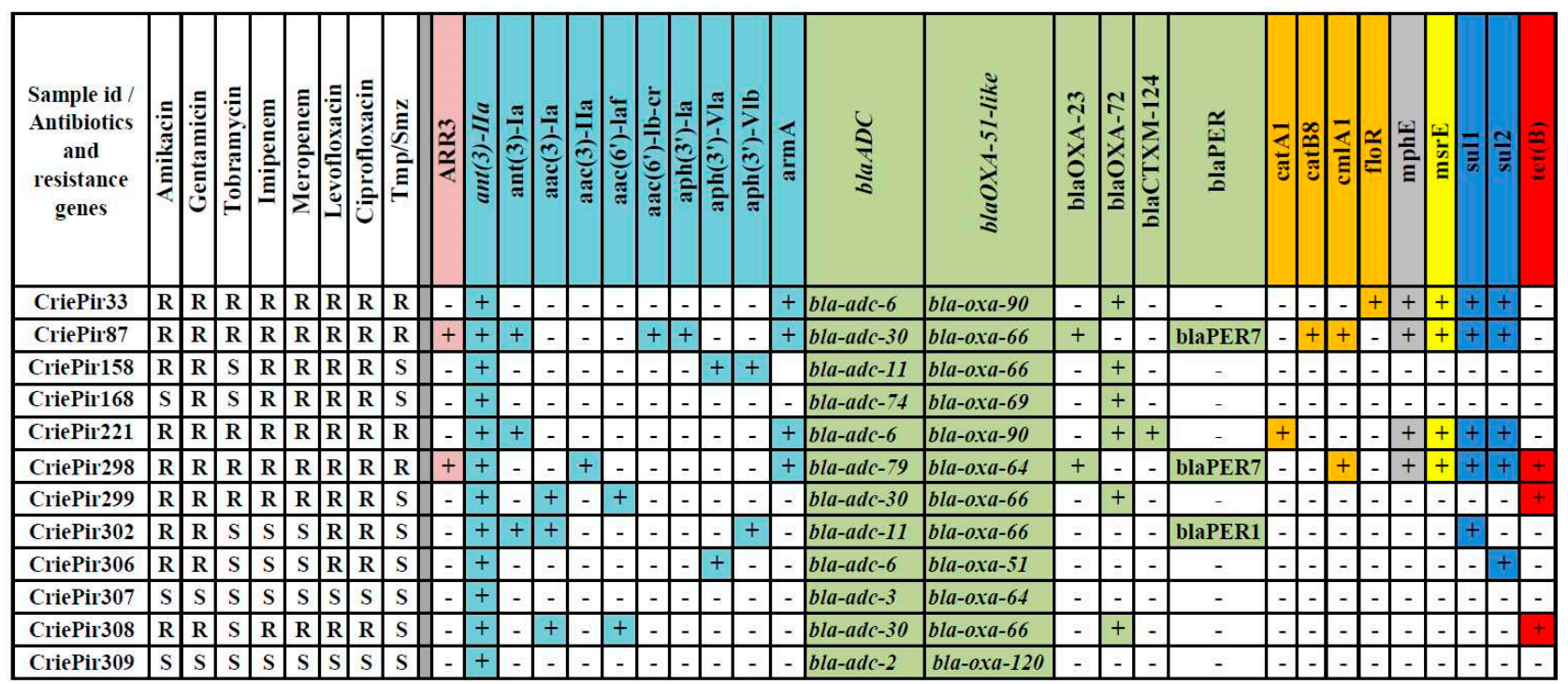

Figure 1. Antibiotic resistance profiles and distribution of antibiotic resistance determinants of clinical A. baumannii isolates studied. Tmp/Smz-trimethoprim/sulfamethoxazole; "+" and "-" indicates the presence and absence of antibiotic resistance determinant, respectively; gene clusters for resistance to different groups of antibiotics are highlighted by different colors. Intrinsic ant(3")-IIa, blaADC and blaOXA-51-like genes are presented at the beginning of corresponding groups and are italicized.

Different alleles of intrinsic cephalosporinase-encoding gene $b l a_{A D C}$ were revealed in all isolates studied. The isolates possessed a wide variety of oxacillinase genes (bla ${ }_{O X A}$, eight genes), most of which encoded carbapenem-hydrolyzing class D $\beta$-lactamases (CHDLs) for those A. baumannii had been previously found to be an original host [24]. CriePir221 also encoded extended spectrum $\beta$-lactamase (ESBL) (bla ${ }_{C T X-M-124}$ gene), while CriePir302 encoded PER-1, and CriePir87 and CriePir298 encoded PER-7 ESBLs, respectively, which were known to possess an increased activity toward broad-spectrum cephalosporins in $A$. baumannii [25]. The isolate CriePir87 was the one having the maximal number of acquired antibiotic resistance genes (13 genes of 23 identified).

Comparative analysis shows that $b l a_{O X A-72}$ and $b l a_{O X A-23}$ represent carbapenemase genes that are likely to confer meropenem and imipenem resistance for 8 out of 12 isolates studied. For the isolates sequenced on MinION, the location of bla $a_{O X A-72}$ was found to be on plasmid (CriePir168), while the blaOXA-23 location was on chromosome (CriePir298). These findings comply with the results described previously [26,27].

\subsection{CRISPR Arrays and CRISPR/Cas Systems}

Ten isolates from our study included confirmed CRISPR repeats sequences determined by CRISPRCasFinder [28]. Three isolates (CriePir168, CriePir298 and CriePir307) carried CRISPR loci with encoded Cas proteins which represent an important component for the functioning of a putative CRISPR/Cas system. It is worth noting that one isolate (CriePir307) having such putative system was susceptible to all antibiotics tested, while the other two isolates were MDR. These three isolates also possessed different integrated plasmid sequences. All other isolates except two (CriePir158 and CriePir299, which did not have any CRISPR spacers) possessed CRISPR arrays but lacked any detectable cas genes. Among them, the isolate CriePir309 draws attention due to the presence of many CRISPR spacers with plasmid localization and the lack of Cas proteins. The highest homology of its spacers was revealed to the following Acinetobacter phage sequences: phiAC-1, vB_AbaM_IME284, AB1, YMC1112R2315 and WCHABP1. The number and characteristics of CRISPR spacers revealed in the isolates studied are presented in Table S2.

Putative CRISPR/Cas systems found in CriePir168, CriePir298 and CriePir307 belonged to Type I-F. Distinct assortment of spacers harbored by each of the three isolate is presented in Figure 2. 


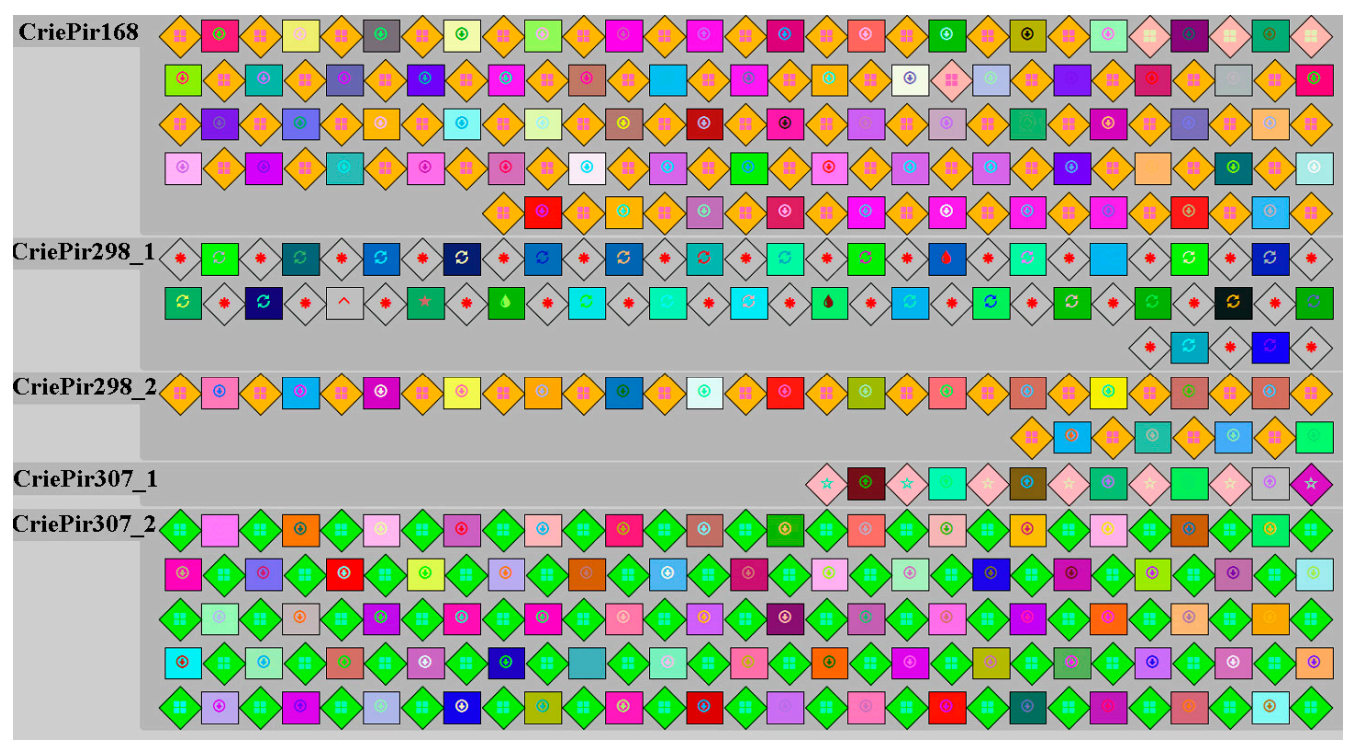

Figure 2. Spacer (squares) and repeat (diamonds) composition for clinical A. baumannii isolates having putative Type I-F CRISPR/Cas systems.

The numbers of spacers in the CRISPR locus of the isolates CriePir168, CriePir298 and CriePir307 are respectively 73, 47 and 85 . Spacer numbers vary without any relationship to plasmid size or the presence/absence of antibiotic resistance genes. Spacer composition varied between isolates. The genome of CriePir168 isolate contains spacers homologous to prophage sequences; the highest homology was found with Acinetobacter phage vB_AbaS_TRS. Prophage sequences comprising CriePir298 CRISPR array included Acinetobacter phages AM106, Ab105-3phi and Pseudomonas phage AUS531phi. The CRISPR spacers of CriePir307 isolate matched with Acinetobacter phages Ab105-2phi, vB_AbaS_TRS1 and YMC1111R3177. The CRISPR arrays analyzed also included plasmid sequences, namely, CriePir168 possessed CRISPR spacers matching Acinetobacter plasmids pDA33382-2-2, pAba7804b and pAB3, the isolate CriePir298 carried spacers homologous to plasmid p2014S01-097-2 and unnamed A. baumannii plasmid of the strain B11911, while CriePir307 was characterized by CRISPR spacers matching unnamed A. baumannii plasmid of the strain CIAT758, pAba9201a, p2014S01-097-2 and pTG31302.

Direct repeats of CriePir168 and CriePir298 belong to structure motif 6 and represent sequence family 8 , while direct repeats of CriePir307 belong to structure motif 4 and represent unclassified sequence family (Figure $\mathrm{S} 1$ ).

The CRISPR/Cas loci of the isolates CriePir168 and CriePir298 were similar and consisted of six genes encoding Cas6 endoribonuclease, three Csy proteins (Csy3, Csy2 and Csy1), Cas3/Cas2 helicase/RNase and Cas1 endonuclease in the vicinity of CRISPR arrays. The CriePir307 isolate carried Cas1, Cas3/Cas2, Cas6, and two Csy proteins (Csy2 and Csy3) downstream of the CRISPR-array. An internal deletion was detected in the locus of this isolate, resulting in the absence of Csy1. None of the genes of described CRISPR/Cas loci contained premature stop codons, and the genes of anti-CRISPR proteins were not found.

Reference isolates, as well as our isolates, were characterized by genotypic antibiotic resistance profiles, plasmid number, the presence of prophage sequences, and CRISPR array type. Maximum likelihood phylogenetic tree of full-length cas1 gene sequences showed that A. baumannii isolates CriePir168 and CriePir298 belonged to Type I-F1 CRISPR/Cas system (30 out of 45 sequences), while CriePir307 belonged to Type I-F2 (15 out of 45 sequences) (Figure 3) [29,30]. Topology of the isolates studied on the phylogenetic trees constructed for full-length cas gene sequences was identical, e.g., the same sequences represented clades containing Type I-F1 and Type I-F2 isolates (Figures S2-S6). For simplicity, the names of our isolates are shortened just to ' $\mathrm{P}$ ' instead of 'CriePir' on these figures. 


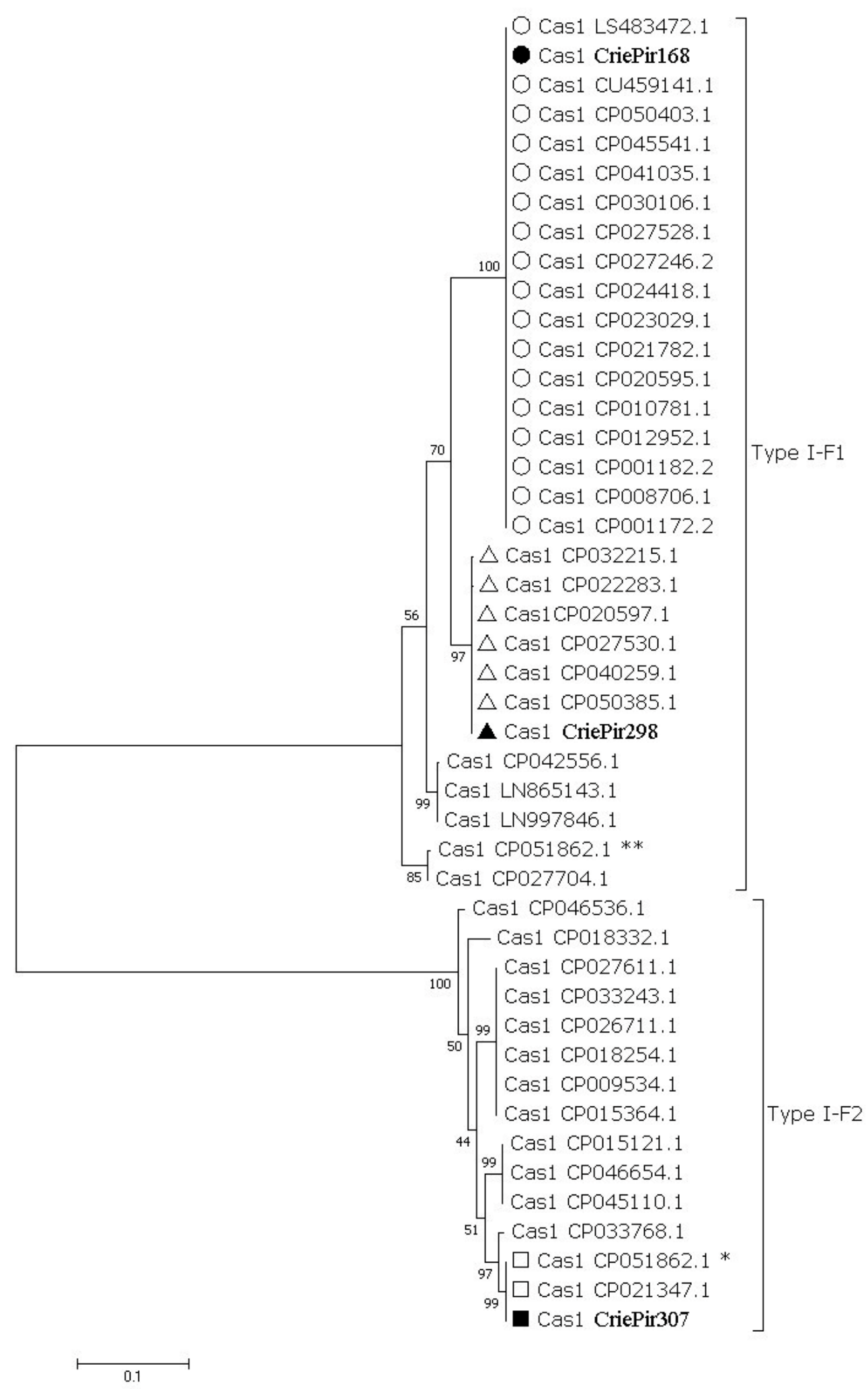

Figure 3. Maximum-likelihood phylogenetic tree of full-length cas1 gene sequences of A. baumannii detected in clinical departments of multidisciplinary medical center in Moscow, Russia during the period of 2017-2019. Bootstrap test (1000 replicates) was used, and bootstrap values are shown at the branch nodes. The sequences identified in this study are marked with black circle, square and triangle, respectively. Reference sequences belonging to the clades containing the sequences identified in this study are marked with transparent circles, squares and triangles, respectively. Sequences identified in this study are indicated by the isolate names, reference sequences-by GenBank accession number. Asterisks $(*, * *)$ indicate two different cassettes of cas genes found in one of the reference isolates. 
To assess the characteristics of A. baumannii CRISPR/Cas system, a list of 242 reference isolates (Table S3) was composed according to CRISPRCasdb. All isolates were divided into four groups according to the CRISPR/Cas system present- "No CRISPR/No Cas", "CRISPR/No Cas", "CRISPR/Cas Type I-F1" and "CRISPR/Cas Type I-F2" (Table S3). To reveal the correlation between $A$. baumannii MDR and CRISPR/Cas system reference isolates, as well as our isolates, were characterized by genotypic antibiotic resistance profiles, plasmid number and the presence of genes encoding virulence factors.

The number of antibiotic resistance genes differs between the analyzed groups of the isolates (Figure 4). The highest number of antibiotic resistance genes (Mean $\pm \mathrm{SD}$, $6.326 \pm 2.466$ ) was found in "No CRISPR/No Cas" A. baumannii isolates, the lowest-in "CRISPR/Cas Type I-F2" isolates (Mean \pm SD, $1.667 \pm 0.976)$. The number of antibiotic resistance genes in "CRISPR/No Cas" and "CRISPR/Cas Type I-F1" did not differ significantly (Figure 4).

Antibiotic resistance genes among the Acinetobacter baumannii isolates

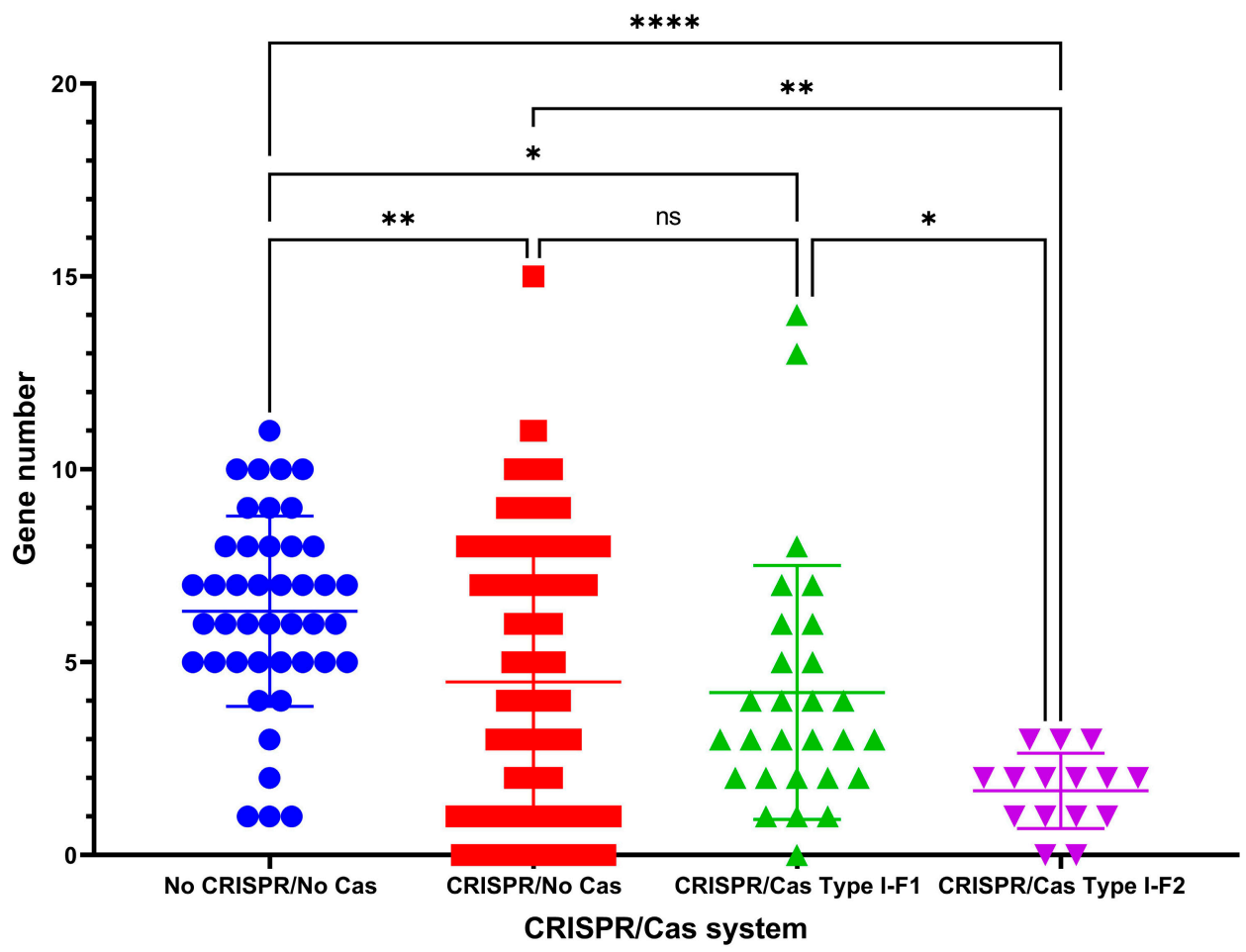

Figure 4. The number of antibiotic resistance genes among A. baumannii isolates. Ns - not significant; $*, * *$ and ${ }^{* * * *}$ indicate the increasing levels of significance.

Interestingly, the lowest number of plasmids was found in "No CRISPR/No Cas" A. baumannii isolates (Mean $\pm \mathrm{SD}, 1.349 \pm 1.378$ ). It differs significantly only from "CRISPR/Cas Type I-F1" group having the highest number of plasmids among the analyzed groups (Mean $\pm \mathrm{SD}, 2.393 \pm 2.132$ ) (Figure S7).

All A. baumannii isolates had similar sets of genes encoding virulence factors. Common set consists of 40 genes: abaI, abaR, adeF, adeG, adeH, bap, barA, barB, basA, basB, basC, basD, basF, basG, basI, basJ, bauA, bauB, bauC, bauD, bauE, bauF, bfmR, bfmS, $\operatorname{csuA}, \operatorname{csuA} / \mathrm{B}, \mathrm{csuB}, \mathrm{csuC}, \mathrm{csuD}$, csuE, entE, ompA, pgaA, pgaB, pgaC, pgaD, plc, and plcD. Significant differences were found in frequencies of occurrence of virulence factors abaI, abaR, bap, basH, bauA, and bfmR (Table S4). AbaI (N-acyl-L-homoserine lactone synthetase) occurs more frequently in "No CRISPR/No Cas" A. baumannii isolates and 
the analyzed groups can be arranged in descending order as "No CRISPR/No Cas" > "CRISPR/No Cas", "CRISPR/Cas Type I-F1" > "CRISPR/Cas Type I-F2". AbaR (DNAbinding HTH domain-containing protein) was found less frequently in "CRISPR/Cas Type I-F2" A. baumannii isolates. It should be noted that "CRISPR/Cas Type I-F1" A. baumannii isolates analyzed in our study completely lack bap gene that encodes biofilm-associated protein. BasH (non-ribosomal peptide biosynthesis thioesterase BasH) was revealed slightly less often in "CRISPR/No Cas" isolates. Moreover, bauA encoding TonB-dependent siderophore receptor BauA was underrepresented in "CRISPR/Cas Type I-F1" group of A. baumannii isolates (in one out of 28 isolates). BfmR (biofilm-controlling response regulator) is the only gene slightly underrepresented in "No CRISPR/No Cas" group (Table S4).

To assess the characteristics of $A$. baumannii putative CRISPR/Cas system containing both CRISPR arrays and cas genes, reference isolates, as well as our isolates, were further characterized. Additionally, the presence of prophage sequences, and CRISPR array types were assessed.

It was found that the isolates possessing Type I-F1 CRISPR/Cas system had significantly more antibiotic resistance genes (Figure 4) and ambiguous prophage sequences in their genomes (Figure 5a). Moreover, Type I-F1 CRISPR/Cas system bearing isolates had significantly less CRISPR4 arrays (the most reliably predicted CRISPRs) (Figure 5b) [31].

Ambiguous prophages among the Acinetobacter baumannii isolates

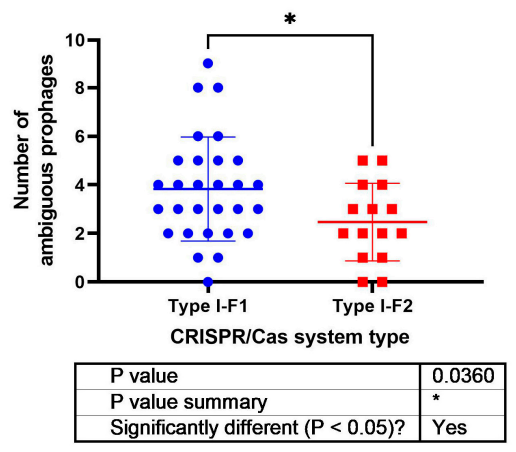

a
CRISPR4 arrays among the Acinetobacter baumannii isolates

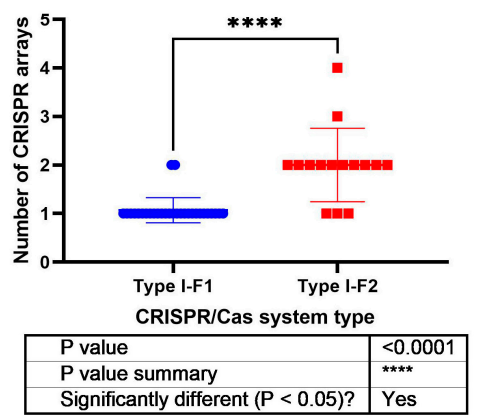

Figure 5. Comparison of $A$. baumannii isolates having different CRISPR/Cas systems by the number of ambiguous prophages (a) and CRISPR4 arrays (b).

It should be noted that no significant differences in plasmid number, active prophage sequence number and low evidence CRISPR array number were detected in the isolates bearing either Type I-F1 or Type I-F2 CRISPR/Cas system (Figures S8-S10). Furthermore, Type I-F1 CRISPR/Cas system of $A$. baumannii had significantly higher number of low evidence CRISPR arrays than CRISPR4 arrays (Figure 6a), and no significant difference was found in active versus ambiguous prophage sequence numbers among them (Figure 6b). At the same time, Type I-F2 CRISPR/Cas system of A. baumannii had significantly more low evidence CRISPR arrays and active prophage sequences (Figures $6 \mathrm{c}$ and $6 \mathrm{~d}$, respectively).

Additionally, we conducted correlation analysis. Normalization of the data sets "Antibiotic resistance genes", "Plasmids", "Active prophages", "Ambiguous prophages", "CRISPR" and "CRISPR4" for both Type I-F1 and Type I-F2 was performed and nonparametric Spearman correlation was calculated, and correlation matrices for each pair of data sets were constructed. It was shown that Type I-F1 CRISPR/Cas systems had moderate positive correlation ( $\mathrm{rs}=0.54, p=0.003$ ) between the number of active prophage sequences and number of antibiotic resistance genes and low negative correlation ( $\mathrm{rs}=-0.44, p=0.02)$ between the number of ambiguous prophage sequences and the number of low evidence CRISPR arrays (Figure 7). 
CRISPR arrays among the Acinetobacter baumannii isolates belonging to CRISPR/Cas Type I-F1

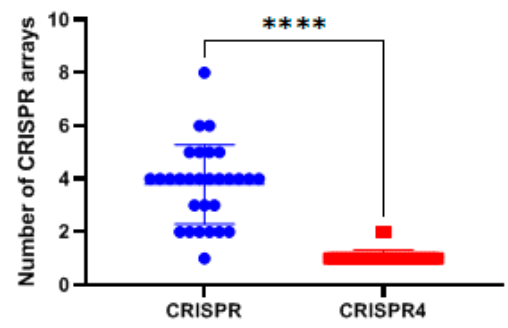

Array type

\begin{tabular}{|l|l|}
\hline $\mathrm{P}$ value & $<0.0001$ \\
\hline $\mathrm{P}$ value summary & $\star \star \star \star$ \\
\hline Significantly different $(\mathrm{P}<0.05)$ ? & $\mathrm{Yes}$ \\
\hline
\end{tabular}

CRISPR arrays among the Acinetobacter baumannii isolates belonging to CRISPR/CasType I-F2

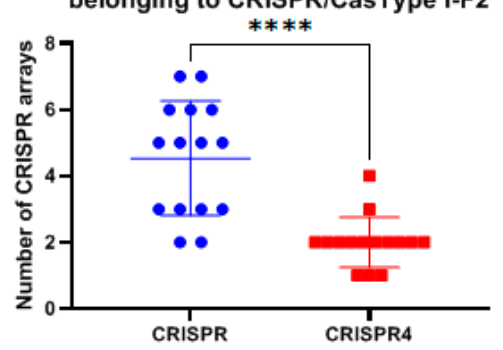

Array type

\begin{tabular}{|l|l|}
\hline $\mathrm{P}$ value & $<0.0001$ \\
\hline $\mathrm{P}$ value summary & $\star \star \star \star$ \\
\hline Significantly differ ent $(\mathrm{P}<0.05)$ ? & Yes \\
\hline
\end{tabular}

Prophages among the Acinetobacter baumannii isolates belonging to CRISPR/Cas Type I-F1

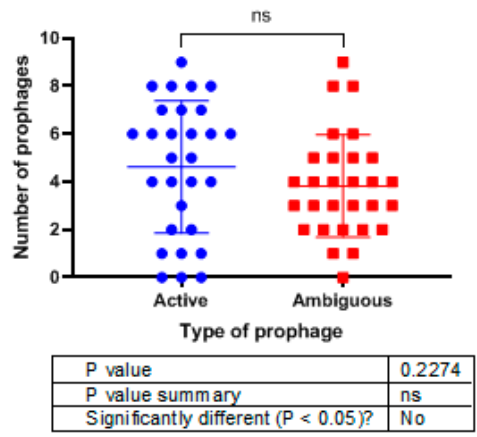

Prophages among the Acinetobacter baumannii isolates belonging to CRISPR/Cas Type I-F2

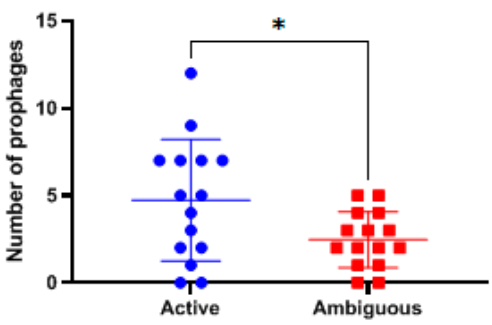

Type of prophage

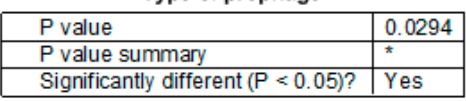

Figure 6. Comparison of A. baumannii isolates carrying Type I-F1 and Type I-F2 systems by CRISPR arrays (a,c) and prophage sequences $(\mathbf{b}, \mathbf{d})$.

TypeI-F1

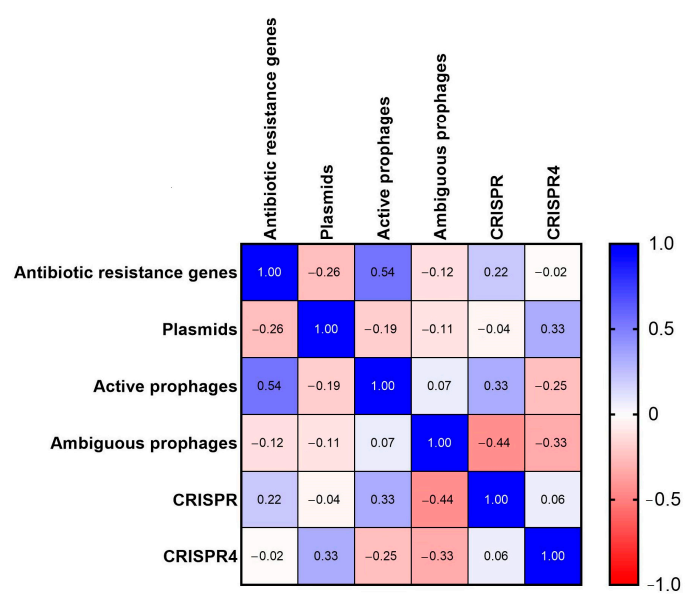

TypeI-F2

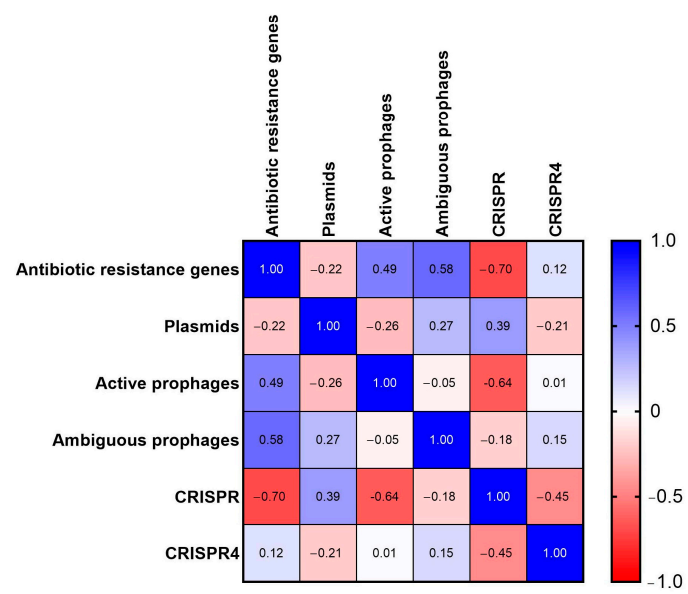

Figure 7. Correlation matrices for Type I-F1 and Type I-F2 CRISPR/Cas system data sets.

Type I-F2 CRISPR/Cas systems had different correlation patterns than those of Type I-F1. Type I-F2 CRISPR/Cas system data sets were characterized by moderate positive correlation ( $\mathrm{rs}=0.58, p=0.025$ ) between the number of ambiguous prophage sequences and the number of antibiotic resistance genes, and by moderate negative correlation between (i) the number of low evidence CRISPR arrays and the number of antibiotic resistance genes (rs $=-0.70, p=0.005$ ) and (ii) the number of low evidence CRISPR arrays and the number of active prophage sequences ( $r s=-0.64, p=0.011$ ) (Figure 6). 


\section{Discussion}

In this study we analyzed 12 clinical isolates of $A$. baumannii which belonged to eight different MLST-based sequence types including the novel ST1487 represented by the isolate CriePir307.We also proposed a hybrid typing scheme (MLST/KL typing) that we recently applied to K. pneumoniae [23] for better distinguishing of the isolates A. baumannii in clinical settings. Although many typing schemes were already provided for pathogenic bacteria, including the ones based on the nucleotide frequency matrices for genomic sequences [32], CRISPR sequences [33], and regular sequences classification [34], the hybrid typing scheme including MLST, KL, and, possibly, OCL loci seems to be the most promising for the species where all of them are available. Additional typing by cgMLST alleles confirmed the classification provided by hybrid typing scheme (Figure S11).

Ten of 12 isolates expressed an MDR phenotype; four of them were resistant to the whole panel of eight antibiotics belonging to various classes. Long-read sequencing on MinION (Oxford Nanopore Technologies, Oxford, UK) allowed to greatly improve the assembly of four A. baumannii genomes (down to one contig for each genome), including the distinguishing of the plasmid sequences. This allowed us to obtain the precise locations of carbapenemase genes for these isolates-bla OXA-72 was found on plasmid for CriePir168, while the blaOXA-23 location was revealed on the chromosome for CriePir298, which complies with previous findings $[26,27]$. In general, the isolates exhibited good compliance between phenotypic and genomic resistance profiles, and no discrepancies were observed for the antibiotics tested. However, the panel was not diverse enough to make global conclusions regarding phenotype-genotype correlations. The data obtained will facilitate future studies of antimicrobial resistance mechanisms in Acinetobacter species.

Additionally, we analyzed CRISPR arrays those were observed in 10 isolates, and putative CRISPR/Cas systems possessed by 3 isolates of our samples and 242 reference isolates according to the CRISPRCasdb. CRISPRs are described in a wide range of prokaryotes [31]. Only $36 \%$ of bacteria carry both CRISPR arrays and cas genes. According to CRISPRCasdb, about $20 \%$ of representatives of Acinetobacter genus and $18 \%$ isolates of A. baumannii species carry both CRISPR arrays and cas genes.

CRISPR/Cas system of Type I is the most widespread in nature [29] and is characterized by the presence of multi-subunit effector complex. Specific compositions of this complex include 9 subtypes, namely, A, B, C, G, D, E, F1 (previously F), F2 (previously F variant), and F3 [29,30]. The most well-known CRISPR/Cas Type I systems are Type I-E systems, while Type I-F1 CRISPR/Cas system is their closest relative [35]. The Type I-F1 CRISPR/Cas system is characterized by a unique fusion of Cas2 to Cas3 (Cas2/3), which together with Cas1 mediate spacer integration into CRISPR locus [36]. Cas2/3 also degrades foreign DNA, which is targeted by the Type I-F1 cascade (i.e., CRISPR-associated complex for antiviral defense) or Csy (i.e., crRNA-guided surveillance) complex. Csy complex typically consists of four different Cas proteins: Csy1 also known as Cas8f, Csy2 (Cas5f), Cas7f (Csy3), and Csy4 (Cas6f) [37]. It should be noted that Csy1 is missing in Type I-F2 CRISPR/Cas system, but the existing Csy2 containing an additional domain may compensate for the roles of Csy1 $[38,39]$.

A. baumannii is known to have Type I-F CRISPR/Cas systems [13], but some species carry type IV variant with genes csf3, csf4 (also known as $\operatorname{din} G$ ) and cas6e, together with CRISPR arrays at both ends [40]. Our study involved A. baumannii isolates detected in clinical departments of multidisciplinary medical center in Moscow, Russia, during the period of 2017-2019, as well as reference isolates having active CRISPR/Cas system according to CRISPRCasdb. Approximately one third of Acinetobacter baumannii species carry both CRISPR arrays and cas genes belonging to Type I-F2. In attempt to reveal specific features of A. baumannii CRISPR/Cas systems, we performed multiparametric analysis of A. baumannii genomes including phylogenetic analysis of full-length cas gene sequences, prophage sequence prediction, genomic antimicrobial resistance profile prediction and detection of CRISPR array types. 
We found the identical topology of the isolates studied on the phylogenetic trees constructed for full-length cas gene sequences, namely, the same sequences represented the clades containing Type I-F1 and Type I-F2 isolates, which implied a vertical spread of the CRISPR/Cas locus in A. baumannii. This fact was also suggested earlier by Karah et al. [13].

In the present study, we have demonstrated clear association of MDR genotype/ phenotype of A. baumannii with the type of its CRISPR/Cas system. Isolates lacking both CRISPR arrays and active cas genes were shown to have much more antibiotic resistance genes than those having only CRISPR arrays or both CRISPR arrays and cas genes. This complies with the fact that CRISPR/Cas system represents a so-called prokaryotic "immune system" that can fight not only against phage infections, but also against dissemination of antibiotic resistance genes in A. baumannii.

Additionally, correlation of A. baumannii virulence factors and CRISPR/Cas systems was found. Virulence factors can be horizontally transferred easily among isolates by phage transduction. Several factors, namely $a b a I, a b a R, b a p$ and $b a u A$, are overrepresented in A. baumannii isolates lacking CRISPR/Cas system. This indicates the role of CRISPR/Cas in fighting against phage infections and preventing horizontal gene transfer.

For the first time the differences between the isolates carrying Type I-F1 and Type I-F2 CRISPR/Cas systems were investigated. One of the most significant differences between Type I-F1 and Type I-F2 isolates of A. baumannii revealed in the present study is the lower number of CRISPR4 arrays in Type I-F1 A. baumannii isolates. Lower number of highly confident CRISPR arrays located together with cas genes suggests that "immune system" of Type I-F2 A. baumannii isolates is stronger. As a result, we noticed another significant difference between Type I-F1 and Type I-F2 isolates of $A$. baumannii, namely, higher number of antibiotic resistance genes in Type I-F1 A. baumannii isolates. This finding may imply that Type I-F1 A. baumannii isolates more easily adapt to environmental conditions through acquisition of antibiotic resistance genes, while Type I-F2 A. baumannii, presumably, may use CRISPR/Cas system to block dissemination of antibiotic resistance genes. It is worth noting that wild type antimicrobial-susceptible E. coli strains carry Type I-F1 CRISPR/Cas system, and it is supposed to interfere with the acquisition of antimicrobial resistance plasmids, maintaining susceptibility in these E. coli isolates [18]. The hypothesis that CRISPR/Cas bacterial adaptive immune systems prevent clinical isolates from acquiring antibiotic resistance elements has been widely discussed; however, it has been described in details for the Enterococci only [40]. In the research mentioned it was suggested that type II CRISPR/Cas systems reduced the acquisition of antibiotic resistance and were negatively associated with MDR isolates. In silico analysis of the pan-genome of 2500 A. baumannii strains by Mangas et al. [41] showed that they could be divided into two groups having different number of common genes within each group, which could be maintained by CRISPR/Cas systems. The group with these defense systems seems to have specific biofilm genes, and would prevent the acquisition of plasmids and, probably, foreign genes, including resistance elements [41].

Moreover, we have shown that Type I-F1 A. baumannii isolates are characterized by the presence of higher number of ambiguous prophage sequences. This fact may also be linked with weakness of their "immune" CRISPR/Cas system, i.e., Type I-F1 A. baumannii isolates include less CRISPR4 arrays, and so they are less capable to fight against phage infections via CRISPR/Cas. Presumably, Type I-F1 A. baumannii isolates possess distinctive molecular mechanisms to arrest phage infections and to convert an active prophage into ambiguous one through point mutations, genome rearrangements, modular exchanges, invasion by mobile DNA elements, massive DNA deletion, etc.

Despite the fact that we had a small collection of clinical isolates of $A$. baumannii, three of 12 isolates were characterized by different types of CRISPR/Cas systems. Their further analysis, together with 242 reference strains, allowed us to find statistically significant differences. For the first time we showed that $A$. baumannii isolates with Type I-F1 and Type I-F2 CRISPR/Cas system are characterized by different numbers of predicted CRISPR4 arrays, antibiotic resistance genes and ambiguous prophage sequences. 
In conclusion, the data obtained could facilitate further investigations in the field of studying the genome dynamics of $A$. baumannii through fast evolving CRISPR/Cas systems, in particular, their role in controlling antibiotic resistance gene transfer and acquisition.

\section{Materials and Methods}

\subsection{Determination of Antibiotic Susceptibility}

All isolates were identified down to a species level by time-of-flight mass spectrometry (MALDI-TOF MS) using the VITEC MS system (bioMerieux, Marcy-l'Étoile, France). The susceptibility was determined by the disc diffusion method using the Mueller-Hinton medium (bioMerieux, Marcy-l'Étoile, France) and disks with antibiotics (BioRad, Marnesla-Coquette, France), and by the boundary concentration method on VITEK2Compact30 analyzer (bioMerieux, Marcy-l'Étoile, France). The isolates were tested for susceptibility/resistance to the following drugs: amikacin, gentamicin, tobramycin, imipenem, meropenem, levofloxacin, ciprofloxacin and trimethoprim/sulfomethoxazole. The panel of antimicrobial compounds included for testing in this study reflected those agents used for human therapy in Russian Federation. To interpret the results obtained, we used the EUCAST clinical breakpoints, version 11.0 (https:/ / www.eucast.org/clinical_breakpoints/, accessed on 20 December 2020) where available.

\subsection{DNA Isolation, Sequencing and Genome Assembly}

Twelve samples were obtained from 11 patients ( 6 males and 5 females) in various sources and clinical departments (Table 1) of multidisciplinary medical center in Moscow, Russia during the period of 2017-2019. Patient age ranged from 27 to 62 with a median equal to 52 years. Ten isolates exhibited MDR phenotypes, and two (CriePir307 and CriePir309) were susceptible to all antibiotics tested.

Genomic DNA was isolated with DNeasy Blood and Tissue kit (Qiagen, Hilden, Germany) and used for paired-end library preparation with Nextera ${ }^{\mathrm{TM}}$ DNA Sample Prep Kit (Illumina ${ }^{\circledR}$, San Diego, CA, USA) and whole-genome sequencing (WGS) of all 12 isolates on Illumina ${ }^{\circledR}$ Hiseq platform (Illumina ${ }^{\circledR}$, San Diego, CA, USA).

WGS was also performed using the Oxford Nanopore MinION sequencing system (Oxford Nanopore Technologies, Oxford, UK) for 4 out of 12 isolates, which carried CRISPR arrays and/or CRISPR/Cas loci. DNA was used to prepare the MinION library with the Rapid Barcoding Sequencing kit SQK-RBK004 (Oxford Nanopore Technologies, Oxford, UK). The amount of initial DNA used for barcoding kit was $400 \mathrm{ng}$ for each sample. All mixing steps for DNA samples were performed by gently flicking the microfuge tube instead of pipetting. All libraries were prepared according to the manufacturer's protocols. The final library was sequenced on R9 SpotON flow cell. The standard $24 \mathrm{~h}$ sequencing protocol was initiated using the MinKNOW software (Oxford Nanopore Technologies, Oxford, UK).

Base calling of the raw MinION data was performed with Guppy Basecalling Software version 3.4.4 (Oxford Nanopore Technologies, Oxford, UK), and demultiplexing was made using Guppy barcoding software version 3.4.4 (Oxford Nanopore Technologies, Oxford, UK). Assemblies were obtained using SPAdes version 3.11 [42] (Illumina sequencing) and Unicycler version 0.4.8-beta [43] (hybrid assemblies).

Genome assemblies, including 8 short-read assemblies and 4 hybrid long- and shortread assemblies, were uploaded to NCBI Genbank under the project number PRJNA687166.

\subsection{Data Processing}

Assembled genomes were processed using custom software pipeline including a set of scripts for seamless integration of various available software tools [44]. The main goals of investigations were to determine the antibiotic resistance in silico, to perform isolate typing using various molecular classification schemes, and to reveal the presence of CRISPR/Cas systems in the genomes of the isolated studied. The parameters useful for epidemiological surveillance and the presence of virulence factors were also studied. We 
used Resfinder 4.0 database for antimicrobial gene identification (https:/ / cge.cbs.dtu.dk/ services/ResFinder/, accessed on 20 December 2020).

Analysis of cgMLST loci was performed using MentaList software (https: / / github. com/WGS-TB/MentaLiST, accessed on 20 December 2020, version 0.2.4), and the tree was build using PHYLOViz online (http: / / online.phyloviz.net, accessed on 20 December 2020).

CRISPRCasFinder [28] was used to identify the presence of CRISPR/Cas systems and spacers in the genomes studied.

Phylogenetic analyses were conducted using Maximum Likelihood (ML) method in MEGA7.0.26 [45]. The statistical significance of the branches was assessed by bootstrap resampling analysis (1000 replicates).

Prophage sequences prediction and evaluation of the probability of a prophage being active was made using Prophage Hunter (https:/ / pro-hunter.genomics.cn/index.php/ Home/Index/index.html, accessed on 20 December 2020) [46].

Direct repeats found in CRISPR arrays were analyzed using CRISPRmapweb tool http: / / rna.informatik.uni-freiburg.de/CRISPRmap/Input.jsp version v1.3.0-2013, accessed on 20 December 2020, since the human samples were routinely collected, and patients' data remained anonymous [47-49].

CRISPR array type was assessed using CRISPRCasdb, where CRISPR4 represents Level 4 CRISPRs (the most reliable ones), while CRISPR levels 1, 2 and 3 may be considered as false CRISPRs [31].

Data analysis and graphing were performed using Prism 9 (GraphPad Software, San Diego, CA, USA).

Supplementary Materials: The following are available online at https:/ /www.mdpi.com/2076-0 $817 / 10 / 2 / 205 /$ s1, Table S1. cgMLST profiles for the isolates studied (pubMLST.org); Table S2. The numbers and features of CRISPR spacers found in A. baumannii isolates from multidisciplinary medical center in Moscow, Russia during the period of 2017-2019; Table S3. A. baumannii reference isolates; Table S4. Differences in virulence factor frequencies among the Acinetobacter baumannii isolates with different CRISPR/Cas systems; Figure S1. Classification of direct repeats of some A. baumannii isolates detected in clinical departments of multidisciplinary medical center in Moscow, Russia during the period of 2017-2019 with CRISPRmap web tool; Figure S2. Maximum-likelihood phylogenetic tree of full-length cas3-cas2 gene sequences of A. baumannii detected in clinical departments of multidisciplinary medical center in Moscow, Russia during the period of 2017-2019; Figure S3. Maximum-likelihood phylogenetic tree of full-length cas 6 gene sequences of A. baumannii detected in clinical departments of multidisciplinary medical center in Moscow, Russia during the period of 2017-2019; Figure S4. Maximum-likelihood phylogenetic tree of full-length csy1 gene sequences of A. baumannii detected in clinical departments of multidisciplinary medical center in Moscow, Russia during the period of 2017-2019; Figure S5. Maximum-likelihood phylogenetic tree of full-length csy2 gene sequences of $A$. baumannii detected in clinical departments of multidisciplinary medical center in Moscow, Russia during the period of 2017-2019; Figure S6. Maximum-likelihood phylogenetic tree of full-length csy3 gene sequences of $A$. baumannii detected in clinical departments of multidisciplinary medical center in Moscow, Russia during the period of 2017-2019; Figure S7. Plasmid number among the A. baumannii isolates; Figure S8. Plasmid number among the A. baumannii isolates with Type I-FI and TypeI-FII CRISPR/Cas systems; Figure S9. Active prophage sequences among the A. baumannii isolates; Figure S10. Low evidence CRISPR arrays among the A. baumannii isolates; Figure S11. cgMLST tree for the isolates studied. The numbers of allele differences are indicated.

Author Contributions: All authors contributed to this study. L.P., V.F. and M.Z. performed the clinical part of the experiment including susceptibility testing; Y.M. and A.P. performed the sequencing part; A.S., M.T., A.P. and A.T. analyzed the data; M.T., Y.M. and A.S. wrote the manuscript; V.A. supervised the project and obtained funding. All authors have read and agreed to the current version of the manuscript. 
Funding: This work was supported by the Ministry of Science and Higher Education of the Russian Federation within the framework of a grant in the form of a subsidy for the creation and development of the «World-class Genomic Research Center for Ensuring Biological Safety and Technological Independence under the Federal Scientific and Technical Program for the Development of Genetic Technologies», agreement No. 075-15-2019-1666.

Informed Consent Statement: Patient consent was waived since the human samples were routinely collected, and patients' data remained anonymous.

Data Availability Statement: The data presented in this study are openly available in NCBI Genbank under the project number PRJNA687166. In addition, publicly available datasets were analyzed in this study. This data can also be found in Genbank (https:/ / www.ncbi.nlm.nih.gov/genbank/, accessed on 20 December 2020). Full list of accession numbers is given in Table S3.

Acknowledgments: The authors thank Yuri Yanushevich for assistance in sample library preparation.

Conflicts of Interest: The authors declare no conflict of interest. The funders had no role in the design of the study; in the collection, analyses, or interpretation of data; in the writing of the manuscript, or in the decision to publish the results.

\section{References}

1. Munoz-Price, L.S.; Weinstein, R.A. Acinetobacter infection. N. Engl. J. Med. 2008, 358, 1271-1281. [CrossRef] [PubMed]

2. Zarrilli, R.; Giannouli, M.; Tomasone, F.; Triassi, M.; Tsakris, A. Carbapenem resistance in Acinetobacter baumannii: The molecular epidemic features of an emerging problem in health care facilities. J. Infect. Dev. Ctries. 2009, 3, 335-341. [CrossRef]

3. World Health Organization. Guidelines for the Prevention and Control of Carbapenem-Resistant Enterobacteriaceae, Acinetobacter Baumannii and Pseudomonas Aeruginosa in Health Care Facilities; WHO: Geneva, Switzerland, 2017.

4. Chebotar, I.V.; Lazareva, A.V.; Masalov, Y.K.; Mikhailovich, V.M.; Mayanskiy, N.A. Acinetobacter: Microbiological, pathogenetic and resistant properties. Vestn. Ross. Akad. Med. Nauk. 2014, 69, 39-50. [CrossRef]

5. Bartual, S.G.; Seifert, H.; Hippler, C.; Luzon, M.A.; Wisplinghoff, H.; Rodriguez-Valera, F. Development of a multilocus sequence typing scheme for characterization of clinical isolates of Acinetobacter baumannii. J. Clin. Microbiol. 2005, 43, 4382-4390. [CrossRef] [PubMed]

6. Diancourt, L.; Passet, V.; Nemec, A.; Dijkshoorn, L.; Brisse, S. The population structure of Acinetobacter baumannii: Expanding multiresistant clones from an ancestral susceptible genetic pool. PLoS ONE 2010, 5, e10034. [CrossRef] [PubMed]

7. van Belkum, A.; Soriaga, L.B.; LaFave, M.C.; Akella, S.; Veyrieras, J.B.; Barbu, E.M.; Shortridge, D.; Blanc, B.; Hannum, G.; Zambardi, G.; et al. Phylogenetic Distribution of CRISPR-Cas Systems in Antibiotic-Resistant Pseudomonas aeruginosa. mBio 2015, 6, e01796-15. [CrossRef]

8. Shariat, N.; Dudley, E.G. CRISPRs: Molecular signatures used for pathogen subtyping. Appl. Environ. Microbiol. 2014, 80, 430-439. [CrossRef]

9. Fabre, L.; Zhang, J.; Guigon, G.; Le Hello, S.; Guibert, V.; Accou-Demartin, M.; de Romans, S.; Lim, C.; Roux, C.; Passet, V.; et al. CRISPR typing and subtyping for improved laboratory surveillance of Salmonella infections. PLoS ONE 2012, 7, e36995. [CrossRef]

10. Kos, V.N.; Deraspe, M.; McLaughlin, R.E.; Whiteaker, J.D.; Roy, P.H.; Alm, R.A.; Corbeil, J.; Gardner, H. The resistome of Pseudomonas aeruginosa in relationship to phenotypic susceptibility. Antimicrob. Agents Chemother. 2015, 59, 427-436. [CrossRef]

11. Turton, J.F.; Wright, L.; Underwood, A.; Witney, A.A.; Chan, Y.T.; Al-Shahib, A.; Arnold, C.; Doumith, M.; Patel, B.; Planche, T.D.; et al. High-Resolution Analysis by Whole-Genome Sequencing of an International Lineage (Sequence Type 111) of Pseudomonas aeruginosa Associated with Metallo-Carbapenemases in the United Kingdom. J. Clin. Microbiol. 2015, 53, 2622-2631. [CrossRef]

12. Di Nocera, P.P.; Rocco, F.; Giannouli, M.; Triassi, M.; Zarrilli, R. Genome organization of epidemic Acinetobacter baumannii strains. BMC Microbiol. 2011, 11, 1-17. [CrossRef]

13. Karah, N.; Samuelsen, O.; Zarrilli, R.; Sahl, J.W.; Wai, S.N.; Uhlin, B.E. CRISPR-cas subtype I-Fb in Acinetobacter baumannii: Evolution and utilization for strain subtyping. PLoS ONE 2015, 10, e0118205. [CrossRef]

14. Horvath, P.; Romero, D.A.; Coute-Monvoisin, A.C.; Richards, M.; Deveau, H.; Moineau, S.; Boyaval, P.; Fremaux, C.; Barrangou, R. Diversity, activity, and evolution of CRISPR loci in Streptococcus thermophilus. J. Bacteriol. 2008, 190, 1401-1412. [CrossRef] [PubMed]

15. Westra, E.R.; Buckling, A.; Fineran, P.C. CRISPR-Cas systems: Beyond adaptive immunity. Nat. Rev. Microbiol. 2014, 12, 317-326. [CrossRef] [PubMed]

16. Ershova, K.; Savin, I.; Kurdyumova, N.; Wong, D.; Danilov, G.; Shifrin, M.; Alexandrova, I.; Sokolova, E.; Fursova, N.; Zelman, V.; et al. Implementing an infection control and prevention program decreases the incidence of healthcare-associated infections and antibiotic resistance in a Russian neuro-ICU. Antimicrob. Resist. Infect. Control. 2018, 7, 1-11. [CrossRef]

17. Zheng, P.X.; Chiang-Ni, C.; Wang, S.Y.; Tsai, P.J.; Kuo, C.F.; Chuang, W.J.; Lin, Y.S.; Liu, C.C.; Wu, J.J. Arrangement and number of clustered regularly interspaced short palindromic repeat spacers are associated with erythromycin susceptibility in emm12, emm75 and emm92 of group A streptococcus. Clin. Microbiol. Infect. 2014, 20, 516-523. [CrossRef] [PubMed] 
18. Aydin, S.; Personne, Y.; Newire, E.; Laverick, R.; Russell, O.; Roberts, A.P.; Enne, V.I. Presence of Type I-F CRISPR/Cas systems is associated with antimicrobial susceptibility in Escherichia coli. J. Antimicrob. Chemother. 2017, 72, 2213-2218. [CrossRef]

19. Li, H.Y.; Kao, C.Y.; Lin, W.H.; Zheng, P.X.; Yan, J.J.; Wang, M.C.; Teng, C.H.; Tseng, C.C.; Wu, J.J. Characterization of CRISPR-Cas Systems in Clinical Klebsiella pneumoniae Isolates Uncovers Its Potential Association with Antibiotic Susceptibility. Front. Microbiol. 2018, 9, 1595. [CrossRef]

20. Arbatsky, N.P.; Shneider, M.M.; Dmitrenok, A.S.; Popova, A.V.; Shagin, D.A.; Shelenkov, A.A.; Mikhailova, Y.V.; Edelstein, M.V.; Knirel, Y.A. Structure and gene cluster of the K125 capsular polysaccharide from Acinetobacter baumannii MAR13-1452. Int. J. Biol. Macromol. 2018, 117, 1195-1199. [CrossRef]

21. Wyres, K.L.; Cahill, S.M.; Holt, K.E.; Hall, R.M.; Kenyon, J.J. Identification of Acinetobacter baumannii loci for capsular polysaccharide (KL) and lipooligosaccharide outer core (OCL) synthesis in genome assemblies using curated reference databases compatible with Kaptive. Microb. Genom. 2020, 6. [CrossRef]

22. Shashkov, A.S.; Cahill, S.M.; Arbatsky, N.P.; Westacott, A.C.; Kasimova, A.A.; Shneider, M.M.; Popova, A.V.; Shagin, D.A.; Shelenkov, A.A.; Mikhailova, Y.V.; et al. Acinetobacter baumannii K116 capsular polysaccharide structure is a hybrid of the K14 and revised K37 structures. Carbohydr. Res. 2019, 484, 107774. [CrossRef] [PubMed]

23. Shelenkov, A.; Mikhaylova, Y.; Yanushevich, Y.; Samoilov, A.; Petrova, L.; Fomina, V.; Gusarov, V.; Zamyatin, M.; Shagin, D.; Akimkin, V. Molecular Typing, Characterization of Antimicrobial Resistance, Virulence Profiling and Analysis of Whole-Genome Sequence of Clinical Klebsiella pneumoniae Isolates. Antibiotics 2020, 9, 261. [CrossRef] [PubMed]

24. Poirel, L.; Naas, T.; Nordmann, P. Diversity, epidemiology, and genetics of class D beta-lactamases. Antimicrob. Agents Chemother. 2010, 54, 24-38. [CrossRef] [PubMed]

25. Bonnin, R.A.; Potron, A.; Poirel, L.; Lecuyer, H.; Neri, R.; Nordmann, P. PER-7, an extended-spectrum beta-lactamase with increased activity toward broad-spectrum cephalosporins in Acinetobacter baumannii. Antimicrob. Agents Chemother. 2011, 55, $2424-2427$. [CrossRef]

26. Wang, H.; Guo, P.; Sun, H.; Wang, H.; Yang, Q.; Chen, M.; Xu, Y.; Zhu, Y. Molecular epidemiology of clinical isolates of carbapenem-resistant Acinetobacter spp. from Chinese hospitals. Antimicrob. Agents Chemother. 2007, 51, 4022-4028. [CrossRef]

27. Jia, H.; Sun, Q.; Ruan, Z.; Xie, X. Characterization of a small plasmid carrying the carbapenem resistance gene bla OXA-72 from community-acquired Acinetobacter baumannii sequence type 880 in China. Infect. Drug Resist. 2019, 12, 1545-1553. [CrossRef]

28. Couvin, D.; Bernheim, A.; Toffano-Nioche, C.; Touchon, M.; Michalik, J.; Neron, B.; Rocha, E.P.C.; Vergnaud, G.; Gautheret, D.; Pourcel, C. CRISPRCasFinder, an update of CRISRFinder, includes a portable version, enhanced performance and integrates search for Cas proteins. Nucleic Acids Res. 2018, 46, W246-W251. [CrossRef]

29. Makarova, K.S.; Wolf, Y.I.; Iranzo, J.; Shmakov, S.A.; Alkhnbashi, O.S.; Brouns, S.J.J.; Charpentier, E.; Cheng, D.; Haft, D.H.; Horvath, P.; et al. Evolutionary classification of CRISPR-Cas systems: A burst of class 2 and derived variants. Nat. Rev. Microbiol. 2020, 18, 67-83. [CrossRef]

30. Koonin, E.V.; Makarova, K.S.; Zhang, F. Diversity, classification and evolution of CRISPR-Cas systems. Curr. Opin. Microbiol. 2017, 37, 67-78. [CrossRef]

31. Pourcel, C.; Touchon, M.; Villeriot, N.; Vernadet, J.P.; Couvin, D.; Toffano-Nioche, C.; Vergnaud, G. CRISPRCasdb a successor of CRISPRdb containing CRISPR arrays and cas genes from complete genome sequences, and tools to download and query lists of repeats and spacers. Nucleic Acids Res. 2020, 48, D535-D544. [CrossRef]

32. Shelenkov, A.; Korotkov, A.; Korotkov, E. MMsat-A database of potential micro- and minisatellites. Gene 2008, 409, 53-60. [CrossRef]

33. Yeh, H.Y.; Awad, A. Genotyping of Campylobacter jejuni Isolates from Poultry by Clustered Regularly Interspaced Short Palindromic Repeats (CRISPR). Curr. Microbiol. 2020, 77, 1647-1652. [CrossRef]

34. Shelenkov, A.; Korotkov, E. Search of regular sequences in promoters from eukaryotic genomes. Comput. Biol. Chem. 2009, 33, 196-204. [CrossRef] [PubMed]

35. Tuminauskaite, D.; Norkunaite, D.; Fiodorovaite, M.; Tumas, S.; Songailiene, I.; Tamulaitiene, G.; Sinkunas, T. DNA interference is controlled by R-loop length in a type I-F1 CRISPR-Cas system. BMC Biol. 2020, 18, 1-16. [CrossRef] [PubMed]

36. Fagerlund, R.D.; Wilkinson, M.E.; Klykov, O.; Barendregt, A.; Pearce, F.G.; Kieper, S.N.; Maxwell, H.W.R.; Capolupo, A.; Heck, A.J.R.; Krause, K.L.; et al. Spacer capture and integration by a type I-F Cas1-Cas2-3 CRISPR adaptation complex. Proc. Natl. Acad. Sci. USA 2017, 114, E5122-E5128. [CrossRef]

37. Rollins, M.F.; Chowdhury, S.; Carter, J.; Golden, S.M.; Wilkinson, R.A.; Bondy-Denomy, J.; Lander, G.C.; Wiedenheft, B. Cas1 and the Csy complex are opposing regulators of Cas2/3 nuclease activity. Proc. Natl. Acad. Sci. USA 2017, 114, E5113-E5121. [CrossRef] [PubMed]

38. Zheng, Y.; Li, J.; Wang, B.; Han, J.; Hao, Y.; Wang, S.; Ma, X.; Yang, S.; Ma, L.; Yi, L.; et al. Endogenous Type I CRISPR-Cas: From Foreign DNA Defense to Prokaryotic Engineering. Front. Bioeng. Biotechnol. 2020, 8, 62. [CrossRef]

39. Pausch, P.; Muller-Esparza, H.; Gleditzsch, D.; Altegoer, F.; Randau, L.; Bange, G. Structural Variation of Type I-F CRISPR RNA Guided DNA Surveillance. Mol. Cell 2017, 67, 622-632.e624. [CrossRef]

40. Palmer, K.L.; Gilmore, M.S. Multidrug-resistant enterococci lack CRISPR-cas. mBio 2010, 1. [CrossRef]

41. Mangas, E.L.; Rubio, A.; Alvarez-Marin, R.; Labrador-Herrera, G.; Pachon, J.; Pachon-Ibanez, M.E.; Divina, F.; Perez-Pulido, A.J. Pangenome of Acinetobacter baumannii uncovers two groups of genomes, one of them with genes involved in CRISPR/Cas defence systems associated with the absence of plasmids and exclusive genes for biofilm formation. Microb. Genom. 2019, 5. [CrossRef] 
42. Bankevich, A.; Nurk, S.; Antipov, D.; Gurevich, A.A.; Dvorkin, M.; Kulikov, A.S.; Lesin, V.M.; Nikolenko, S.I.; Pham, S.; Prjibelski, A.D.; et al. SPAdes: A new genome assembly algorithm and its applications to single-cell sequencing. J. Comput. Biol. 2012, 19, 455-477. [CrossRef] [PubMed]

43. Wick, R.R.; Judd, L.M.; Gorrie, C.L.; Holt, K.E. Unicycler: Resolving bacterial genome assemblies from short and long sequencing reads. PLoS Comput. Biol. 2017, 13, e1005595. [CrossRef]

44. Shelenkov, A.; Petrova, L.; Fomina, V.; Zamyatin, M.; Mikhaylova, Y.; Akimkin, V. Multidrug-Resistant Proteus mirabilis Strain with Cointegrate Plasmid. Microorganisms 2020, 8, 1775. [CrossRef] [PubMed]

45. Kumar, S.; Stecher, G.; Tamura, K. MEGA7: Molecular Evolutionary Genetics Analysis Version 7.0 for Bigger Datasets. Mol. Biol. Evol. 2016, 33, 1870-1874. [CrossRef] [PubMed]

46. Song, W.; Sun, H.X.; Zhang, C.; Cheng, L.; Peng, Y.; Deng, Z.; Wang, D.; Wang, Y.; Hu, M.; Liu, W.; et al. Prophage Hunter: An integrative hunting tool for active prophages. Nucleic Acids Res. 2019, 47, W74-W80. [CrossRef]

47. Alkhnbashi, O.S.; Costa, F.; Shah, S.A.; Garrett, R.A.; Saunders, S.J.; Backofen, R. CRISPRstrand: Predicting repeat orientations to determine the crRNA-encoding strand at CRISPR loci. Bioinformatics 2014, 30, i489-i496. [CrossRef]

48. Lange, S.J.; Alkhnbashi, O.S.; Rose, D.; Will, S.; Backofen, R. CRISPRmap: An automated classification of repeat conservation in prokaryotic adaptive immune systems. Nucleic Acids Res. 2013, 41, 8034-8044. [CrossRef]

49. Raden, M.; Ali, S.M.; Alkhnbashi, O.S.; Busch, A.; Costa, F.; Davis, J.A.; Eggenhofer, F.; Gelhausen, R.; Georg, J.; Heyne, S.; et al. Freiburg RNA tools: A central online resource for RNA-focused research and teaching. Nucleic Acids Res. 2018, 46, W25-W29. [CrossRef] [PubMed] 